\title{
The magnetic susceptibility in QCD
}

\author{
Claudio Bonati* \\ Dipartimento di Fisica dell'Università di Pisa and INFN - Sezione di Pisa, Largo Pontecorvo 3, \\ I-56127 Pisa, Italy \\ E-mail: bonati@df.unipi.it
}

\section{Massimo D’Elia}

Dipartimento di Fisica dell'Università di Pisa and INFN - Sezione di Pisa, Largo Pontecorvo 3, I-56127 Pisa, Italy

E-mail: delia@df.unipi.it

\section{Marco Mariti}

Dipartimento di Fisica dell'Università di Pisa and INFN - Sezione di Pisa, Largo Pontecorvo 3, I-56127 Pisa, Italy

E-mail: mariti@df.unipi.it

\section{Francesco Negro}

Dipartimento di Fisica dell'Università di Genova and INFN - Sezione di Genova, Via Dodecaneso 33, I-16146 Genova, Italy

E-mail: fnegro@ge.infn.it

\section{Francesco Sanfilippo ${ }^{\dagger}$}

Laboratoire de Physique Théorique (Bat. 210) Université Paris SUD, F-91405 Orsay-Cedex,

France

E-mail: francesco.sanfilippodth.u-psud.fr

Recently much work has been devoted to the study of QCD coupled to a background magnetic field. Strongly interacting matter acts as a magnetic medium and it is natural to study the properties of this medium, in particular to understand if it behaves like a diamagnetic or a paramagnetic material. A serious difficulty in studying these properties by means of LQCD simulations is the quantization of the magnetic field in a toroidal geometry. We will expose a method to overcome this difficulty and we will present data obtained for the $N_{f}=2$ theory that show that the QCD medium is paramagnetic.

31st International Symposium on Lattice Field Theory - LATTICE 2013

July 29 - August 3, 2013

Mainz, Germany

\footnotetext{
*Speaker.

${ }^{\dagger}$ Now at: School of Physics and Astronomy, University of Southampton, Southampton SO17 1BJ, United Kingdom.
} 


\section{Introduction}

Magnetic field of intensities ranging from $10^{10}$ to $10^{15-16} \mathrm{~T}$ are expected to be present in such disparate environments as compact astrophysical objects (for magnetars see $e . g$ [1]), the early Universe (see e.g [2]) and heavy ion collisions (see e.g. [3]). Since a magnetic field of intensity $10^{15-16} \mathrm{~T}$ corresponds to $|e| B \sim 1 \mathrm{GeV}^{2}$, it is clear that its interaction can significantly affect the properties of strongly interacting matter. As a consequence, in the last few years the interplay between strong and electromagnetic interactions have received much attention (for a comprehensive review see the volume [4]).

For ordinary materials, the computation of the reaction to an external magnetic field is a standard problem of condensed matter physics. For non ferromagnetic media and small magnetic fields, the induced polarization is linear in the intensity of the external field and a quantitative measure of the reaction of the material is the magnetic susceptibility. It appears natural to ask the same type of fundamental questions for the strongly interacting matter: does it react linearly to external magnetic fields? If this is the case, what is the value of its magnetic susceptibility? In particular, is it a paramagnetic or a diamagnetic medium? Despite the simplicity and clear-cut nature of these questions, it is nontrivial to answer them.

The standard tool for studying non-perturbative aspects of QCD dynamics is the lattice formulation of the theory and it is not difficult to add an external magnetic field to the discretized theory. However, in a toroidal geometry (the one usually adopted in simulations to reduce finite size effects), the magnetic field values are not arbitrary but get quantized. Intuitively this is related to the fact that when applying the Stokes theorem on a compact manifold without boundary the result must be independent of the surface used for the flux computation. To enforce this independence we have to impose a relation between the admissible magnetic fluxes and the smallest electrical charge present in the theory. In the QCD case the smallest charge is $q=|e| / 3$ and, assuming $\mathbf{B}=B \hat{\mathbf{z}}$ and a $3 D$ toroidal manifold, one gets the quantization condition [5]

$$
|e| B=6 \pi b /\left(\ell_{x} \ell_{y}\right)
$$

where $\ell_{x}, \ell_{y}$ are the periods of the torus in the $x, y$ directions and $b \in \mathbb{Z}$. This quantization condition is the main obstruction to a simple lattice answer to the previous questions.

In the following we will present the method developed in [6] to overcome these difficulties and the results obtained by applying it to the case of $N_{f}=2$ staggered fermions: strongly interacting matter at finite temperature behaves as a linear paramagnetic medium and, near deconfinement, its magnetic susceptibility is of the same order of magnitude of that characterizing typical strongly paramagnetic ordinary materials (like e.g liquid oxygen).

\section{The method}

The magnetic susceptibility and, more generally, all the magnetic properties of a (homogeneous) medium, are related to the change of the free energy density $f=F / V$ in presence of an external magnetic field:

$$
\Delta f(B, T)=-\frac{T}{V} \log \left(\frac{Z(B, T, V)}{Z(0, T, V)}\right)
$$


where $Z=\exp (-F / T)$ is the partition function. Since free energies are notoriously difficult to compute by means of numerical simulations, the standard procedure to evaluate magnetic susceptibilities in condensed matter simulations is to study the expectation value of the second derivative of $f$, which is a much better behaved observable than the magnetic free energy density. This is however not possible in the present setting, since the magnetic field is quantized and, as a consequence, derivatives with respect to $B$ of Eq. (2.1) are not well defined.

The basic idea of the method introduced in [6] is to extract the magnetic susceptibility and the other magnetic properties directly from the behaviour of free energy differences $f\left(b_{2}\right)-f\left(b_{1}\right) \equiv$ $f\left(B_{2}\right)-f\left(B_{1}\right)$ (where $b_{2}$ and $b_{1}$ are integers), which are computed by using the elementary formula

$$
f\left(b_{2}\right)-f\left(b_{1}\right)=\int_{b_{1}}^{b_{2}} \frac{\partial f(b)}{\partial b} \mathrm{~d} b
$$

with the integrand function being evaluated on a grid of points in the interval $\left[b_{1}, b_{2}\right]$ (grid that have to be fine enough for the errors associated to the numerical integration to be under control). In order to follow this strategy we have to analytically continue the function $f(b)$, which is properly defined only for $b \in \mathbb{Z}$, on the whole real axis, which is done in the following way.

An external magnetic field is introduced in Lattice QCD simulations by adding to the $S U(3)$ links variables $U_{\mu}(n)$ the non dynamical $U(1)$ phases $u_{\mu}(n)$ associated to the magnetic field, i.e. with the replacement $U_{\mu}(n) \rightarrow u_{\mu}(n) U_{\mu}(n)$. A simple choice for the $U(1)$ phases corresponding to $\mathbf{B}=B \hat{\mathbf{z}}$ is e.g.

$$
\begin{array}{ll}
u_{y}^{(q)}(n)=e^{i a^{2} q B n_{x}} & \left(=e^{i 2 \pi b n_{x} /\left(L_{x} L_{y}\right)} \text { for the } u \text { flavor }\right) \\
\left.u_{x}^{(q)}(n)\right|_{n_{x}=L_{x}}=e^{-i a^{2} q L_{x} B n_{y}} & \left(=e^{-i 2 \pi b n_{y} / L_{y}} \text { for the } u \text { flavor }\right)
\end{array}
$$

and $u_{\mu}^{(q)}(n) \equiv 1$ otherwise. In this expression $q$ is the charge of the considered flavour, $L_{x}, L_{y}$ are the lattice extents in the $x, y$ directions, $a$ is the lattice spacing and $1 \leq n_{\mu} \leq L_{\mu}$. The magnetic field in the $\hat{z}$ direction associated to the phases Eq. (2.3) is uniform only if the quantization condition Eq. (1.1) is respected, otherwise a singularity analogous to a Dirac sting is present in the continuum limit. The analytical continuation required for the application of Eq. (2.2) is obtained by removing the requirement that $b \in \mathbb{Z}$ in the expressions in Eq. (2.3). Since we work on finite lattices the free energy density $f(b)$ is then an analytic function of $b$ and Eq. (2.2) can be safely applied.

We emphasize that $\partial f(b) / \partial b$ defined in such a way is not related in any direct way to the magnetization of the system: the value of $\partial f(b) / \partial b$ (also for integer $b$ ) depends on the analytical continuation adopted and it is thus devoid of any intrinsic physical value. Its only use is to be integrated to extract the free energy finite differences through Eq. (2.2), which are independent of the analytical continuation used (for an explicit numerical check see [6]) and are physically meaningful as far as $b_{1}$ and $b_{2}$ are integers.

Once $\Delta f(B, T)$ has been computed by using Eq. (2.2) we have to properly renormalize it, in order to allow for a smooth continuum limit extrapolation. The only divergences that do not cancel in the difference $\Delta f$ are the $B$-dependent ones and it can be show that such divergences are temperature independent (see e.g. [7, 8]). Motivated by this result and by the physical observation that we are interested in the magnetic properties of the thermal medium and not in those of the 
vacuum, we adopted the renormalization prescription

$$
\Delta f_{R}(B, T)=\Delta f(B, T)-\Delta f(B, 0) .
$$

From the behavior of $\Delta f_{R}(B, T)$ for small fields it is possible to verify that the medium is linear and, eventually, to extract the value of its magnetic susceptibility by using

$$
\Delta f_{R} \simeq-\frac{\tilde{\chi}}{2 \mu_{0}} \mathbf{B}^{2} \equiv-\frac{\hat{\chi}}{2}(e \mathbf{B})^{2} .
$$

$\tilde{\chi}$ is related to the standard SI magnetic susceptibility by the relation $\chi=\tilde{\chi} /(1-\tilde{\chi})$ and it is used in order to properly take into account the fact that in our simulations the medium has no back-reaction on the magnetic field (see [6] for more details). The equivalent of $\tilde{\chi}$ in natural units is $\hat{\chi}$ defined by the last equality in Eq. (2.5) and the relation between the two susceptibilities is simply $\hat{\chi} \simeq 10.9 \tilde{\chi}$.

\section{Numerical results}

The method described in the previous section has been applied in [6] to the study of the magnetic properties of $N_{f}=2 \mathrm{QCD}$. The theory was discretized by using the standard rooted staggered formulation and, although $m_{u}=m_{d}$, isospin symmetry is explicitly broken by the interaction with the magnetic field, since $q_{u}=2|e| / 3$ and $q_{d}=-|e| / 3$.

To use Eq. (2.2) we need to measure the observable

$$
M \equiv a^{4} \frac{\partial f}{\partial b}=\frac{1}{4 L_{t} L_{x} L_{y} L_{z}} \sum_{q=q_{u}, q_{d}}\left\langle\operatorname{tr}\left\{\frac{\partial D^{(q)}}{\partial b} D^{(q)^{-1}}\right\}\right\rangle,
$$

where $D^{(q)}$ is the Dirac matrix of the charge $q$ fermion and $L_{\mu}$ is the lattice extent in the $\mu$ direction. $M$ was evaluated by means of a noisy estimator, using for each measure 10 random vectors. Measures have been performed on $\mathscr{O}\left(10^{3}\right)$ configurations generated by the usual RHMC algorithm for each value of the parameters used, i.e. for values of the pion mass in the range $200-480 \mathrm{MeV}$ and for several values of the lattice spacing (for more details see Tab. 1 of [6]). As our reference $T=0$ value for the renormalization subtraction we used the result obtained on symmetric lattices.

An example of the results obtained for $M$ is shown in the left panel of Fig. (1). The oscillations in the results are a clear signal of the unphysical nature of $M$ and are related to the presence of the unphysical string when $b$ is not an integer. Two different harmonics are visible in the result, which can be associated to the $u$ and $d$ contributions to $M$. Oscillations are nevertheless smooth enough for the result to be numerically integrated. The integration, using 16 determination of $M$ in each quantum, is performed by using a spline interpolation and a bootstrap analysis is used to evaluate the numerical error. Several tests have been performed by using different integration schemes, spline interpolations and number of $M$ determinations; in all the cases compatible result are obtained, which shows that the integration procedure is very stable (see the Supplementary Material of [6] for more details).

Assuming the relation $a^{4} \Delta f=c_{2} b^{2}+\mathscr{O}\left(b^{4}\right)$ to hold true for integer $b$ values, a convenient way to extract the coefficient $c_{2}$ is to study the differences

$$
a^{4}(f(b)-f(b-1)) \equiv \int_{b-1}^{b} M(\tilde{b}) \mathrm{d} \tilde{b} \simeq c_{2}(2 b-1) .
$$



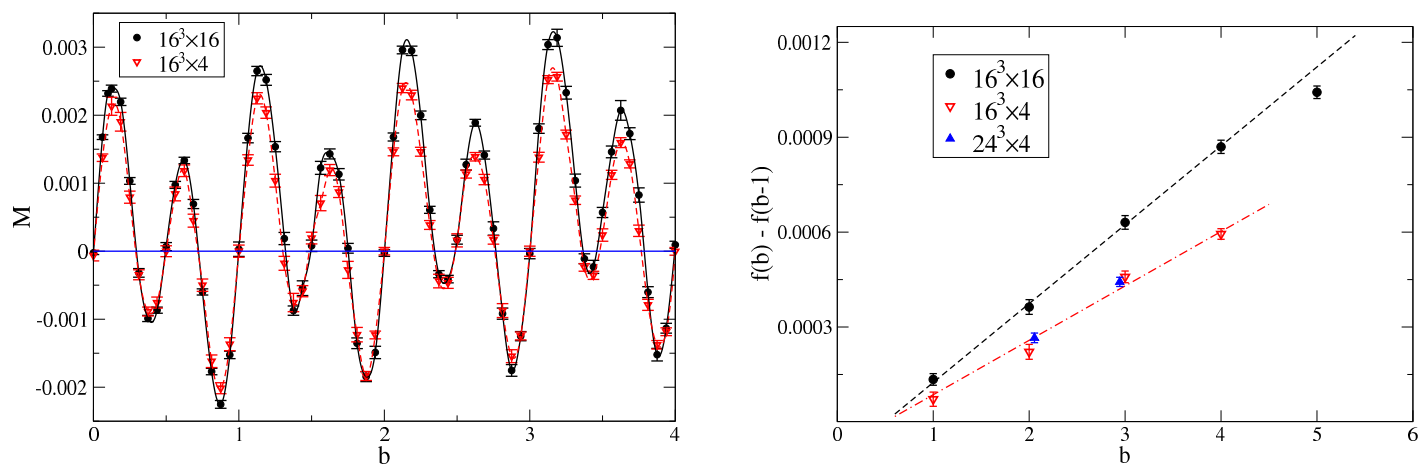

Figure 1: Some results obtained with lattice spacing $a \approx 0.188 \mathrm{fm}$ and pion mass $m_{\pi} \approx 480 \mathrm{MeV}$. (left) $M$ computed on $16^{4}$ and $16^{3} \times 4$ lattices together with third order spline interpolations; (right) $f(b)-f(b-1)$ computed on $16^{4}, 16^{3} \times 4$ and $24^{3} \times 4$ lattices together with the linear fit explained in the text.

This is convenient since in this way we do not need to compute $\partial f(b) / \partial b$ on the whole $[0, b]$ interval but only on some quanta. This strategy also presents the advantage that the integration error does not correlate the measures on different quanta, whose estimates are thus statistically independent of each other.

Some data for these free energy differences, together with fits according to Eq. (3.2), are shown in the right panel of Fig. (1). From this figure it can be seen that the fit nicely works for small enough magnetic field, and thus the strongly interacting medium is linear, while for greater $b$ values deviations from Eq. (3.2) are visible. In all the cases we limited ourself to the study of the leading linear term, which is the one needed to extract the magnetic susceptibility.

By following this strategy both for the finite $T$ and the $T=0$ data we arrive to the relation $a^{4} \Delta f_{R}=c_{2 R} b^{2}+\mathscr{O}\left(b^{4}\right)$, where $c_{2 R}=c_{2}(T)-c_{2}(T=0)$. The last step needed to extract the magnetic susceptibility is just a conversion into physical units:

$$
\tilde{\chi}=-\frac{|e|^{2} \mu_{0} c}{18 \hbar \pi^{2}}\left(L_{x} L_{y}\right)^{2} c_{2 R} \quad \hat{\chi}=-\frac{1}{18 \pi^{2}}\left(L_{x} L_{y}\right)^{4} c_{2 R}
$$

The data obtained for $\tilde{\chi}$ are shown in Fig. (2) (for the numerical data see Tab. 1 of [6]) and, since $|\tilde{\chi}| \ll 1$, we have $\chi \approx \tilde{\chi}$, where $\chi$ is the usual magnetic susceptibility in SI units.

Fig. (2) displays several interesting features. First of all we notice that data do not show any significant dependence on the lattice spacing and only slightly depend on the value of the pion mass. The magnetic susceptibility is everywhere non negative, so we have shown that strongly interacting matter at finite temperature behaves as a paramagnetic medium. Moreover the value of the magnetic susceptibility in the explored range is of the same order of magnitude of that of strongly paramagnetic ordinary materials, like e.g liquid oxygen. Another interesting feature of Fig. (2) is the strong increasing of the magnetic susceptibility in the neighbourhood of the deconfinement crossover, which for the masses used in this work is located in the range $160-170 \mathrm{MeV}$ : in the low temperature phase, data are much smaller that the ones in the deconfined phase, and in fact they are compatible with zero within errors. 


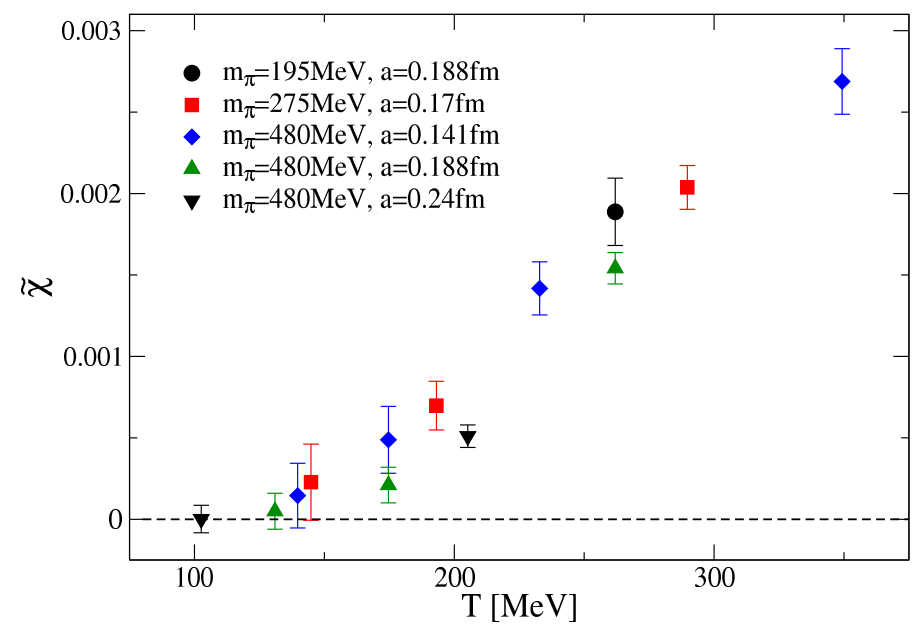

Figure 2: Final results for the $\tilde{\chi}$ magnetic susceptibility in SI units.

\section{Discussion and conclusions}

In this proceeding we presented the method introduced in [6] to study the magnetic properties of the finite temperature strongly interacting matter and the first results obtained by applying it to the case of $N_{f}=2$ staggered fermions. This method is theoretically well founded and completely non-perturbative, all the systematic errors can be analyzed independently and they turned out to be well under control (see [6] for more details). The results obtained by means of other approaches (see [9] and $[10,11]$ ) give a qualitatively similar picture of the dependence of the magnetic susceptibility on the temperature.

The most natural extension of the numerical results presented in this proceeding is the use of improved discretizations and physical quark masses. This has been done in [12] by using $2+1$ flavors, a tree-level Symanzik improved action for the gauge fields, a stout smearing improvement for the staggered fermions and physical values for the $u, d$ and $s$ masses. Higher values of the magnetic susceptibility are obtained in this new setting, but the main features of Fig. (2) remains unaltered. It was however possible to obtain a better signal to noise ratio in the confided phase and, in particular, to explicitly display the paramagnetic behaviour of the low temperature phase. These results are in good quantitative agreement with the analogous ones reported in [11]. Of particular phenomenological relevance could be the observation that, near deconfinement, the magnetic contribution to the pressure is a relevant fraction $\left(\sim 15 \%\right.$ for $|e| B \sim 0.1 \mathrm{GeV}^{2}, \sim 50 \%$ for $\left.|e| B \sim 0.2 \mathrm{GeV}^{2}\right)$ of the thermal contribution (see [12]) and could possibly induce even-by-event fluctuations (see [11]).

Acknowledgements: We thank E. D'Emilio, E. Fraga and S. Mukherjee for useful discussions. Numerical computations have been performed on computer facilities provided by INFN, in particular on two GPU farms in Pisa and Genoa and on the QUONG GPU cluster in Rome. 


\section{References}

[1] R. C. Duncan and C. Thompson, Astrophys. J. 392, L9 (1992).

[2] T. Vachaspati, Phys. Lett. B 265, 258 (1991). D. Grasso and H. R. Rubinstein, Phys. Rept. 348, 163 (2001) [astro-ph/0009061].

[3] V. Skokov, A. Y. Illarionov and V. Toneev, Int. J. Mod. Phys. A 24, 5925 (2009) [arXiv:0907.1396 [nucl-th]]. V. Voronyuk, V. D. Toneev, W. Cassing, E. L. Bratkovskaya, V. P. Konchakovski and S. A. Voloshin, Phys. Rev. C 83, 054911 (2011) [arXiv:1103.4239 [nucl-th]]. A. Bzdak and V. Skokov, Phys. Lett. B 710, 171 (2012) [arXiv:1111.1949 [hep-ph]]. W. -T. Deng and X. -G. Huang, Phys. Rev. C 85, 044907 (2012) [arXiv:1201.5108 [nucl-th]].

[4] D. Kharzeev, K. Landsteiner, A. Schmitt and H. -U. Yee, Lect. Notes Phys. 871, 1 (2013).

[5] G. 't Hooft, Nucl. Phys. B 153, 141 (1979). J. Smit and J. C. Vink, Nucl. Phys. B 286, 485 (1987). P. H. Damgaard and U. M. Heller, Nucl. Phys. B 309, 625 (1988). M. H. Al-Hashimi and U. J. Wiese, Ann. Phys. 324, 343 (2009) [arXiv:0807.0630 [quant-ph]].

[6] C. Bonati, M. D’Elia, M. Mariti, F. Negro and F. Sanfilippo, Phys. Rev. Lett. 111, 182001 (2013) [arXiv:1307.8063 [hep-lat]].

[7] G. S. Bali, F. Bruckmann, G. Endrodi, Z. Fodor, S. D. Katz, S. Krieg, A. Schafer and K. K. Szabo, JHEP 1202, 044 (2012) [arXiv:1111.4956 [hep-lat]].

[8] G. S. Bali, F. Bruckmann, G. Endrodi, F. Gruber and A. Schaefer, JHEP 1304, 130 (2013) [arXiv:1303.1328 [hep-lat]].

[9] L. Levkova and C. DeTar, arXiv:1309.1142 [hep-lat] and these proceedings.

[10] G. S. Bali, F. Bruckmann, G. Endrodi and A. Schafer, these proceedings [arXiv:1310.8145 [hep-lat]].

[11] G. S. Bali, F. Bruckmann, G. Endrodi and A. Schafer, arXiv:1311.2559 [hep-lat].

[12] C. Bonati, M. D’Elia, M. Mariti, F. Negro and F. Sanfilippo, arXiv:1310.8656 [hep-lat]. 